FEITOZA; Daniela de Souza; FREITAS; Maria Célia de; SILVEIRA; Rita Edna da - Traumatismo crânio- 223 encefálico: diagnósticos de enfermagem a vítimas atendidas em UTI. Revista Eletrônica de Enfermagem, $v$. 06, n. 02, p. 223-233, 2004. Disponível em www.fen.ufg.br

\title{
TRAUMATISMO CRÂNIO-ENCEFÁLICO: DIAGNÓSTICOS DE ENFERMAGEM A VÍTIMAS ATENDIDAS EM UTI
}

CRANIOCEREBRAL TRAUMA: NURSING DIAGNOSIS FOR VICTIMS ASSISTANCE ON INTESIVE CARE UNITY.

ATENDIDAS EN UNIDADES DE TERAPIA INTENSIVA

\section{Daniela de Souza Feitoza ${ }^{1}$ Maria Célia de Freitas ${ }^{2}$ Rita Edna da Silveira ${ }^{2}$}

\begin{abstract}
RESUMO:O processo de enfermagem possibilita um cuidado individualizado aos clientes, caracterizando um trabalho autônomo e assistência de qualidade. Objetivou-se Identificar diagnósticos de Enfermagem em pacientes vítimas de traumatismos crânio-encefálico (TCE) e elaborar um modelo de implantação de cuidados, através da taxonomia NANDA, utilizando-se o raciocínio de Risner. Realizou-se estudo descritivo-exploratório, retrospectivo aos pacientes internados no ano 2002, tipo estudo de caso, hospital de referência em traumatologia, em Fortaleza-Ce. Coletou-se os dados nos livros de registro das UTIS I II, III, IV e, Centro de Processamento de dados, nos meses de janeiro a março de 2003. Contabilizou-se 143 (100\%) paciente internados. Sobressaíam-se os TCE com 57 (40\%) dos casos, seguido dos politraumatismos com TCE com 30 (21\%) e traumatismos raqui-medular 11 (7,5\%). Torna-se importante implementar o processo de enfermagem o que permite planejar a assistência, otimizar tempo e garantir a qualidade do cuidado.
\end{abstract}

PALAVRAS-CHAVES: Traumatismo Craniocerebral; Diagnóstico de Enfermagem; Qualidade dos cuidados de Saúde.

SUMMARY: The enfermagem process makes possible a care individualized the customers, characterizing an autonomous work and quality attendance. It was aimed at to Identify diagnoses of Enfermagem in patient traumatismos victims cranium-encefálico (TCE) and to elaborate a model of implantation of cares, through the taxonomia NANDA, being used the reasoning of Risner. He/she/you took place descriptive-exploratory study, retrospective to the patients interned in the year 2002, type case study, reference hospital in traumatologia, in Fortaleza-Ce. It was collected the data in the books of registration of UTIs I II, III, IV and, data processing Center, the months of January to March of 2003. It was counted $143(100 \%)$ patient interned. They were stood out TCE with 57 $(40 \%)$ of the cases, followed by the politraumatismos with TCE with $30(21 \%)$ and traumatismos raqui-medular 11 (7,5\%). He/she/you becomes important to implement the enfermagem process that allows to plan the attendance, to optimize time and to guarantee the quality of the care.

KEY WORD: Craniocerebral Trauma; Nursing Diagnosis; Quality of Health Care.

RESUMEN: El proceso de enfermería posibilita un cuidado individualizado a clientes, caracterizando una atención autónoma con un trabajo de calidad. La pesquisa fue dirigida para identificar diagnostico de enfermería al paciente victima de trauma cráneo - encefalico (TCE) y para elaborar un modelo de la implantación de cuidados, con el taxonomía NANDA, siendo utilizado el razonamiento de Risner. Fue realizado un estudio descriptivo-exploratorio, retrospectivo a los pacientes internados en el año 2002, tipo estudio de caso en un Hospital de referencia en traumatologia, en Fortaleza-Ce. Los dados fueran recogidos en los libros del registro de UTI I II, III, IV y, del Centro de Procesamiento de datos, no periodo de enero a marzo de 2003. Fueran identificados 143 (100\%) pacientes internados. Destacó los TCE con 57 (40\%) de los casos, seguido por los politraumatizados con TCE con 30 (21\%) y $11(7.5 \%)$ traumatismos raqui-medular. Por tanto es importante poner el proceso de enfermería en ejecución e esto permite planear la atención, optimizar tiempo y para garantizar la calidad del cuidado.

\footnotetext{
${ }^{1}$ Enfermeira, graduada pela Univesidade Federal do Ceará, Coordenadora do Serviço de Epidemiologia da Secretaria Municipal de Saúde de Itapipoca-Ce, com especialização em Enfermagem em Terapia Intensiva pela Universidade vale do Acaraú-Ce e Vigilância à saúde e Epidemiologia pela Universidade Federal do Ceará. e-mail para Contato: danielsfeitoza@bol.com.br ou danielsfeitoza6@hotmail.com. Endereço para contato: Rua Espanha, $n^{\circ} 200$, Bairro Vila Pery, Fortaleza-CE, CEP 60721210. Fone: (0xx85) 292.8398.

${ }^{2}$ Enfermeira, graduada pela Universidade Estadual do Ceará, Professora do Curso de Enfermagem da Universidade Estadual do Ceará e Enfermeira do Instituto Dr. Jóse Frota (Hospital de Referência em Traumatologia, Fortaleza-CE
} 
FEITOZA; Daniela de Souza; FREITAS; Maria Célia de; SILVEIRA; Rita Edna da - Traumatismo crânio- 224 encefálico: diagnósticos de enfermagem a vítimas atendidas em UTI. Revista Eletrônica de Enfermagem, $v$. 06, n. 02, p. 223-233, 2004. Disponível em www.fen.ufg.br

PALABRAS CLAVES: Trauma Craneocerebral; Diagnostico de Enfermería; Calidad de la Atención de Salud.

\section{INTRODUÇÃO}

Durante muitos anos a enfermagem foi vista como uma profissão meramente tecnicista, aspecto este fortalecido por muito tempo pelas próprias escolas de enfermagem, que procuravam atender as necessidades de um mercado em contínuo crescimento e carente em mão de obra estritamente especializada.

Aos poucos, com o avanço da ciência, as próprias escolas de enfermagem sentem a necessidade de se mudar esse paradigma, inicia-se então investimentos no processo de valorização do raciocínio clínico dessa categoria. Nesse contexto surge o processo de enfermagem trazendo novas possibilidades de se organizar a prática e contribuindo para uma maior autonomia do profissional e qualidade da assistência.

Desse modo, o estudo propõe a implantação da sistematização da assistência de Enfermagem em Unidade de Terapia Intensiva, cuja clientela são pacientes vítimas de Traumas.

A idéia surgiu a partir da vivencia em estágio do curso de especialização de enfermagem em unidade de terapia intensiva, em uma das unidades do Instituto Dr. Jose Frota (IJF). Na ocasião realizei estudo clínico com paciente vítima de trauma raquimedular, no qual utilizei o raciocínio de Risner para identificar diagnósticos de enfermagem e traçar o planejamento com avaliação somativa e formativa.

Desse modo, a pretensão deste Estudo é dar continuidade ao trabalho anterior, porém, com tema central voltado à aplicação do processo de Enfermagem a paciente vítima de Traumatismo crânio encefálico.

Os traumatismos crânio encefálico (TCE) são freqüentes no Brasil, em sua maioria são causados pelos acidentes de trânsito, mergulhos em águas rasas, agressões, quedas e projéteis de armas de fogo. De uma maneira geral a gravidade das lesões esta relacionada com a intensidade do trauma, pois mesmos leves podem produzir lesões graves.

Aproximadamente 100.000 brasileiros, morrem vitimados por trauma a cada ano, e cerca de um milhão e quinhentos são feridos em acidentes (ARRUDA, 2000)

A Organização Mundial de Saúde (OMS) avalia que uma de cada quatro a nove pessoas, nos países em desenvolvimento, sofre a cada ano de lesões incapacitantes e que $2 \%$ do total da população mundial esta incapacitada como resultado de lesões ocasionadas por acidentes ou violência (PEIXOTO, 1998).

Com base nos dados do Sistema de Informações Hospitalares do SUS (Sistema Único de Saúde) de 2000, A mortalidade hospitalar ficou com $3,9 \%$ do total de internações. $\mathrm{O}$ índice foi maior entre as vítimas do sexo masculino $(3,1 \%)$ e as mortes tiveram maior incidência na faixa dos 14 aos 19 anos.

Durante o ano de 2002, segundo dados obtidos no Instituto José Frota, hospital de referencia para traumas em Fortaleza-Ce, foram admitidos nas UTIs, 673 (100\%) pacientes, destes $198(29,4 \%)$ evoluíram para óbito e 475 (70,5\%) receberam alta. A média de permanência de internação foi maior a 14,5 dias.

Nesse contexto acredita-se que a normatização da assistência, o planejamento das ações e a implantação do Processo de Enfermagem contribuiriam para melhoria da qualidade da assistência a esses pacientes, bem como possibilitaria uma assistência mais qualificada.

Segundo ARRUDA (2000, p.02), "o processo de Enfermagem vem dar suporte e orientar o profissional na elaboração de diagnósticos e intervenções de enfermagem". Os diagnósticos direcionam as intervenções de forma rápida, objetiva e precisa. O processo de Enfermagem atua como um instrumento 
FEITOZA; Daniela de Souza; FREITAS; Maria Célia de; SILVEIRA; Rita Edna da - Traumatismo crânio- 225 encefálico: diagnósticos de enfermagem a vítimas atendidas em UTI. Revista Eletrônica de Enfermagem, $v$. 06, n. 02, p. 223-233, 2004. Disponível em www.fen.ufg.br

metodológico, que quia o enfermeiro sobre o que diagnosticar, intervir e avaliar.

Apesar da importância e garantia de cuidados oferecidos por uma equipe multidisciplinar capacitada, é necessário garantirmos também a qualidade dos serviços.

Considera-se que nas Unidades de Terapia Intensiva, o enfermeiro ofereça assistência contínua a pacientes críticos, e isso exige desse profissional uma melhor capacitação, fundamentação teórica para aplicar o raciocínio clínico e diagnóstico. Mas é necessário que todas essas informações sobre o estado do cliente sejam coletadas, registradas, sistematizadas em diagnósticos, implementadas e avaliadas.

Portanto, fica claro que é imprescindível a implementação do processo de enfermagem nesse contexto. Uma vez que se torna necessário o uso instrumento que auxilie o enfermeiro em UTI a avaliar o paciente vítima de trauma, em suas condições clínicas e iniciar intervenções de enfermagem de forma sistematizada.

Haja vista que o processo de Enfermagem é tido como uma atividade que norteia de forma lógica e coerente às ações de enfermagem, A coleta de dados é fundamentada nos conhecimentos de uma teoria de enfermagem o que o torna uma atividade de cunho científico para os enfermeiros.

Sabe-se que, muitas vezes, os enfermeiros que atuam na prática cotidiana hospitalar e até nas unidades de saúde têm encontrado dificuldades variadas em implementar o processo de enfermagem e identificação de diagnósticos com elaboração de um planejamento de ações. Acredita-se que, talvez pela filosofia das próprias instituições que sobrecarrega o profissional com atividades burocráticas, e o mesmo não consegue organizar espaço que o permita desenvolver estudos, favorecendo o esclarecimento de dúvidas e obtenção de certeza que a sua utilização no cuidado, do dia-a-dia, revelará a diferença na transparência e definição de suas ações na equipe multidisciplinar.

Assim sendo, constituem-se como objetivos: Identificar os diagnósticos de Enfermagem em pacientes vítimas de traumatismos crânio-encefálico, internados nas UTIs de um hospital do município de Fortaleza$\mathrm{Ce}$, estabelecer um perfil dos diagnósticos de Enfermagem dos pacientes vítimas de TCE e Elaborar um modelo de implantação de cuidados, através da taxonomia North American Nursing Diagnosis Association (NANDA I), utilizando o raciocínio de RISNER (1990).

\section{PROCEDIMENTO METODOLÓGICO}

Estudo descritivo- exploratório, tipo estudo de caso, realizado em Hospital de grande porte da rede SUS, no município de Fortaleza-Ce, unidade de referência em traumatologia. Realizado no período de janeiro a março de 2003. Utilizou-se o Estudo de Caso por permitir a junção de aspectos semelhantes, passando a ser representativo para uma dada população em Estudo. Segundo LUDKE \& ANDRE (1986), seu desenvolvimento compreende três fases:

Fase exploratória: Visa selecionar e delimitar os pontos ou questões a serem estudadas do objeto de estudo e localizar as fontes de dados necessários a pesquisa.

Delimitação do Estudo: uma vez delimitado os pontos a serem estudados, procede-se a coleta dos dados, utilizando-se instrumentos estruturados.

Análise e elaboração do relatório: visa analisar as informações, elaborar relatórios e gerar conclusões.

Dessa forma, optou-se por trabalhar com pacientes vítimas de TCE por ser o tipo de trauma mais freqüente naquelas unidades.

Iniciou-se a coleta dos dados somente após a autorização do Comitê de ética, da diretoria da instituição e chefia de Enfermagem das UTIs. 
FEITOZA; Daniela de Souza; FREITAS; Maria Célia de; SILVEIRA; Rita Edna da - Traumatismo crânio- 226 encefálico: diagnósticos de enfermagem a vítimas atendidas em UTI. Revista Eletrônica de Enfermagem, $v$.

06, n. 02, p. 223-233, 2004. Disponível em www.fen.ufg.br

A coleta de dados foi realizada nos livros de registro das Unidades II, III e IV que contém informações sobre: data da admissão, diagnóstico, sexo, data de saída da unidade por óbito ou alta para enfermaria. Coletou-se alguns dados também no CPD (Centro de Processamento de dados).

A população constituiu-se por todos os atendimentos realizados a pessoas no ano de 2002, admitidas nas UTIs II, III e IV (adultos e idosos) independente de idade ou sexo. Sendo a amostra constituída exclusivamente por pacientes vítimas de TCE, que após atendimento em Emergência ou enfermaria, foram encaminhados para uma daquelas unidades.

Após a coleta os dados foram analisados e organizados em tabelas e gráficos para caracterizar a população no ano de 2002, segundo sexo, tipos de traumas mais freqüente e média de tempo de permanência nas Unidades.

Em seguida, Elaborou-se estudo de caso, a partir de informações contidas em prontuário, com paciente daquela unidade, aplicando-se o raciocínio de Risner, com elaboração dos principais diagnósticos de Enfermagem e planejamento das ações de enfermagem com implementação, estabelecimento de metas e objetivos para os principais diagnósticos identificados.

\section{RESULTADOS E DISCUSSÃO}

\section{Caracterização das vítimas}

Os 143 pacientes em Estudo, foram admitidos em uma das unidades de terapia intensiva do IJF e permaneceram por um período médio de 11 a 40 dias. De acordo com os registros das unidades prevaleceram os seguintes tipos de traumas: TCE, politraumatismo + TCE, TRM, politraumatismo e trauma de abdômen. Dos 143 pacientes, 52 $(36,4 \%)$ evoluíram a óbito e 91 (63,6\%) receberam alta sendo admitidos em outras unidades. Constatou-se que o maior número de óbitos ocorreu em pacientes vítimas de TCE, representando um total de $23(44,2 \%)$ dos óbitos. Esse resultado é bastante significativo, evidenciando que os TCE representam a principal causa de morbidade e mortalidade nos grandes centros urbanos (CINTRA, 2000).

TABELA 01- Distribuição numérica e percentual de pacientes internados nas

UTIs II, III e IV segundo sexo e tipo e traumas. Fortaleza, 2002.

\begin{tabular}{lccc}
\multicolumn{1}{c}{ TIPO DE TRAUMA } & \multicolumn{3}{c}{ SEXO } \\
\cline { 2 - 4 } & $\mathbf{M}$ & $\mathbf{F}$ & TOTAL \\
\hline & $\mathbf{N}^{\circ}(\%)$ & $\mathbf{N}^{\circ}(\%)$ & $\mathbf{N}^{\circ}(\%)$ \\
TCE & $\mathbf{5 0}(87,7$ & $\mathbf{0 7 1}(2,3)$ & $\mathbf{5 7}(40,0)$ \\
POLITRAMATISMO+TCE & $\mathbf{2 6}(86,5)$ & $\mathbf{0 4}(13,5)$ & $\mathbf{3 0}(21,0)$ \\
TRM & $\mathbf{1 0}(91,0)$ & $\mathbf{0 1}(9,0)$ & $\mathbf{1 1}(7,5)$ \\
POLITRAUMATISMO & $\mathbf{3 3}(89,0)$ & $\mathbf{0 4}(11,0)$ & $\mathbf{3 7}(2,6)$ \\
TRAUMA DE ABDÓMEM & $\mathbf{0 7}(87,5)$ & $\mathbf{0 1}(12,5)$ & $\mathbf{0 8}(5,5)$ \\
\hline TOTAL & $\mathbf{1 2 6}(88,0)$ & $\mathbf{1 7}(12,0)$ & $\mathbf{1 4 3 1 0 0}$ \\
\hline
\end{tabular}

De acordo com a tabela 01 dos 143 pacientes, 126 (88\%) eram homens e 17 (12\%) mulheres, o que revela que os homens se expõem mais a riscos, talvez pelo estilo de vida ou ao contexto sócio cultural em que estão inseridos. Observamos ainda que os TCE, predominaram tanto no sexo masculino com 50 
FEITOZA; Daniela de Souza; FREITAS; Maria Célia de; SILVEIRA; Rita Edna da - Traumatismo crânio- 227 encefálico: diagnósticos de enfermagem a vítimas atendidas em UTI. Revista Eletrônica de Enfermagem, $v$. 06, n. 02, p. 223-233, 2004. Disponível em www.fen.ufg.br

(88\%) dos casos, como no sexo feminino com $07(12 \%)$

GRÁFICO I-Distribuição percentual de pacientes admitidos nas UTIS II, III e IV segundo o tipo de trauma. Fortaleza, 2002

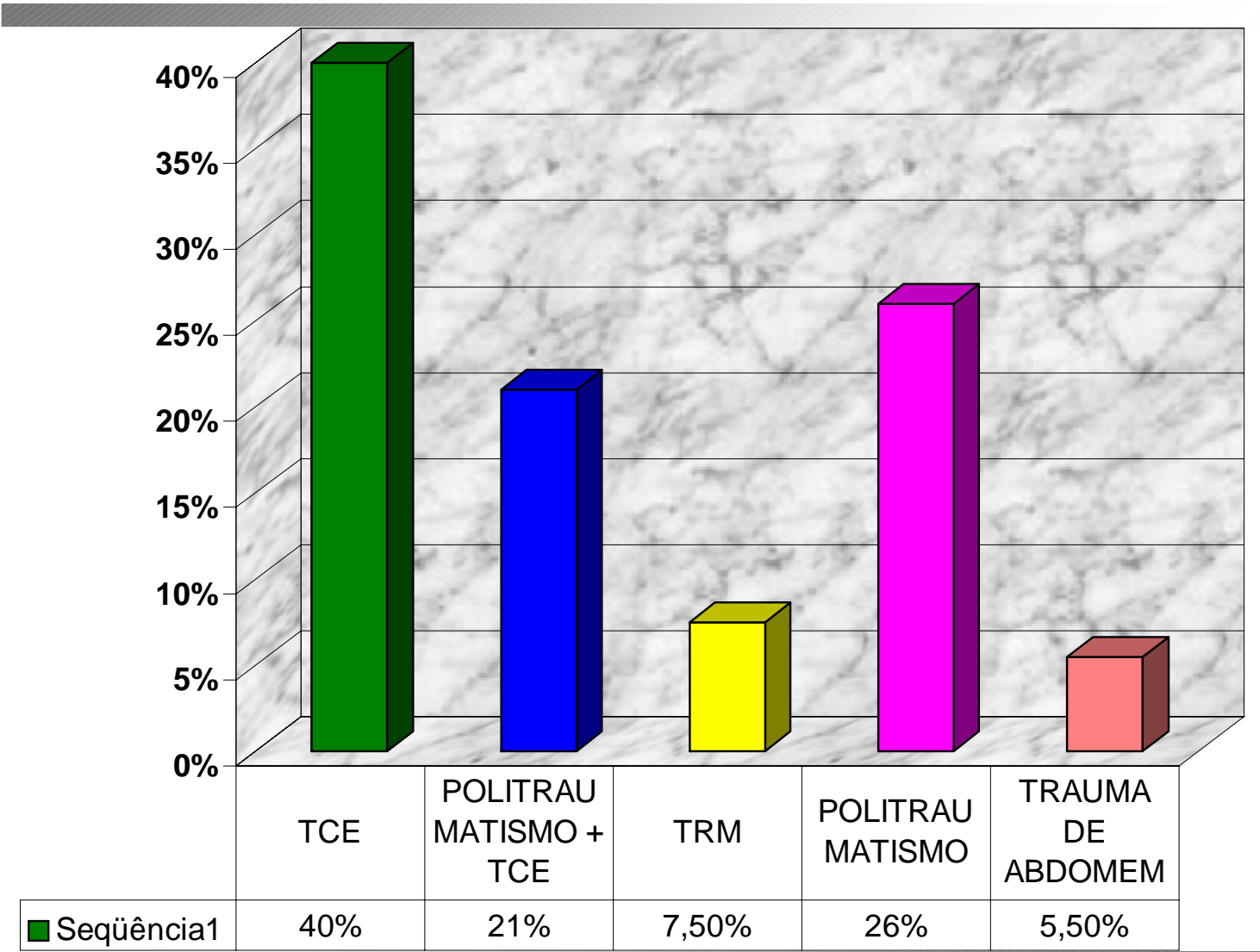

Ao se analisar o gráfico I, observa-se que os Traumatismos Crânio encefálico representaram 57 (40\%) do total das vítimas admitidas nas unidades, sendo superados apenas pelos politraumatismos com 37 (26\%) dos pacientes. Em seguida predominaram os politraumatismos seguidos de TCE com 30
(21\%) das vítimas. De acordo com CINTRA (2000), quase $50 \%$ dos pacientes politraumatizados apresentam TCE associado. Os Traumatismos raqui medular (TRM), representaram $11(7,55)$, enquanto os traumas de abdômen 08 (5,5\%) do total dos traumas. 
FEITOZA; Daniela de Souza; FREITAS; Maria Célia de; SILVEIRA; Rita Edna da - Traumatismo crânio- 228 encefálico: diagnósticos de enfermagem a vítimas atendidas em UTI. Revista Eletrônica de Enfermagem, $v$. 06, n. 02, p. 223-233, 2004. Disponível em www.fen.ufg.br

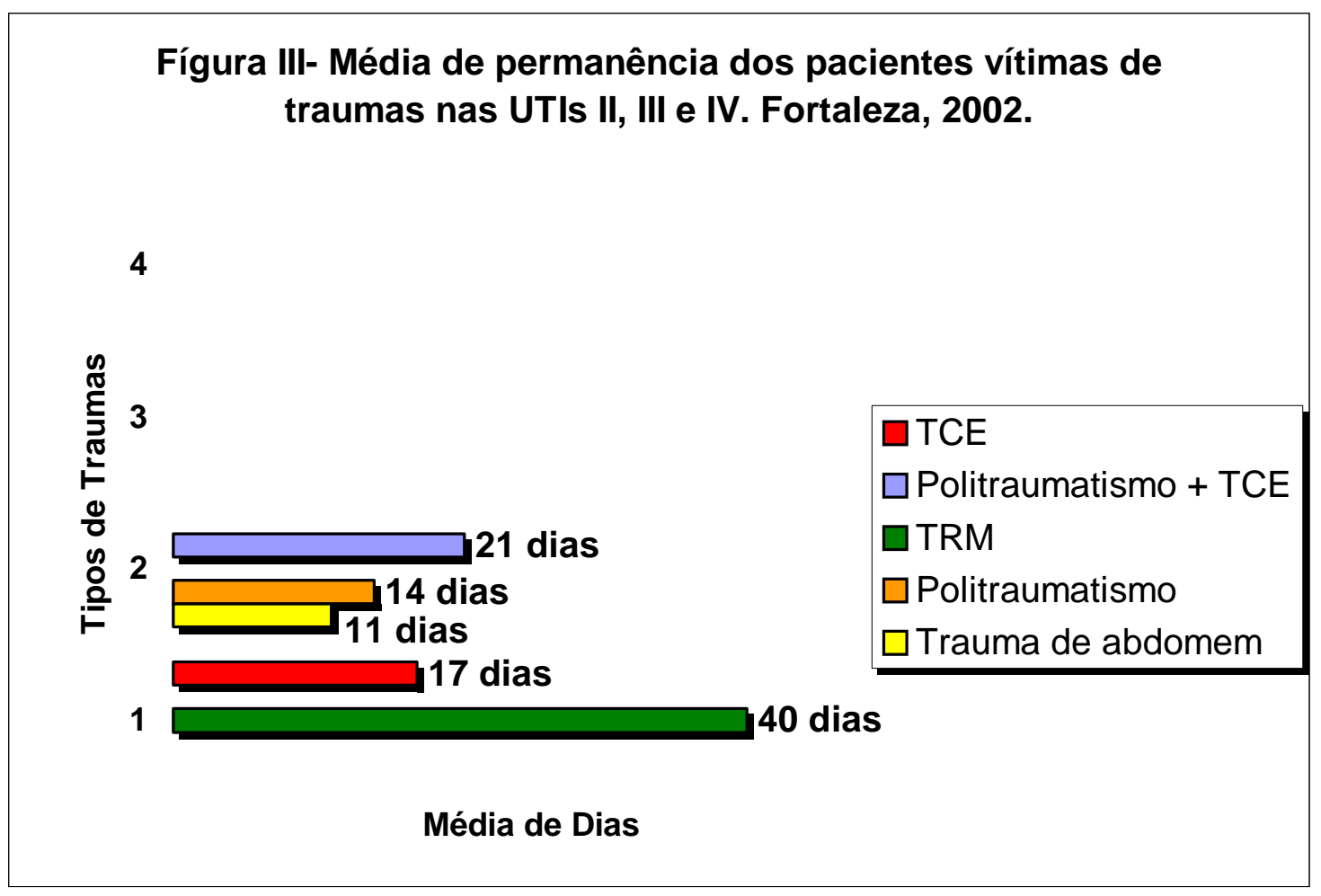

A figura III mostra a média em dias de permanência nas UTIs desses pacientes. Observa-se que as vítimas de TRM permaneceram em média 40 dias, o elevado tempo de permanência talvez esteja relacionado com as complicações respiratórias e as lesões medulares alta (CINTRA, 2000). Em seguida aparecem os politraumatismos associados a TCE com média de permanência de 21 dias. Os pacientes vítimas de TCE permaneceram aproximadamente 17 dias, politraumatizados 14 dias e as vítimas de trauma de abdômen 11 dias. É importante ressaltar que as doenças neurológicas têm seu processo de recuperação lento, requer uma internação prolongada e sujeita a complicações (BRUNNER e SUDDARTH, 1990). Os dados nos permitem refletir sobre a necessidade da aplicação do processo de Enfermagem nas UTIs e conseqüentemente do planejamento da assistência (intervenções) de acordo com os diagnósticos de enfermagem. A sistematização da assistência através da utilização dos diagnósticos de enfermagem proporciona uma visão global das condições em que cada paciente se encontra, otimizando o tempo, garantindo uma assistência individualizada e de qualidade e diminuindo as seqüelas.

\section{Aplicando o raciocínio Diagnóstico}

Selecionou-se o raciocínio de Risner como "caminho" para formulação de Diagnósticos de enfermagem (D.E). RISNER (1990) enfoca o processo de enfermagem dividindo-o em duas etapas:

$1^{\text {a }}$ etapa: composta pelas fases de análise dos dados e síntese:

- $\quad$ Análise dos dados $\rightarrow$ nessa fase examina-se os dados coletados quanto à congruência ou lacunas;

- Síntese $\rightarrow$ Agrupam-
se os dados em padrões,
comparando-se estes a conceitos,
literatura; faz-se inferências ou
geração de hipóteses e elabora-se
causas relacionadas a inferências.


FEITOZA; Daniela de Souza; FREITAS; Maria Célia de; SILVEIRA; Rita Edna da - Traumatismo crânio- 229 encefálico: diagnósticos de enfermagem a vítimas atendidas em UTI. Revista Eletrônica de Enfermagem, $v$. 06, n. 02, p. 223-233, 2004. Disponível em www.fen.ufg.br

$2^{\mathrm{a}}$ etapa: diz respeito à construção redacional de diagnósticos, a partir de uma taxonomia. Neste estudo como já mencionamos se utilizará a taxonomia de NANDA, em que os diagnósticos constituem-se de:

\section{categorias diagnósticas + fatores relacionados + características definidoras.}

Após terem sido analisados os dados foram agrupados em padrões e a síntese foi elaborada a partir do modelo abaixo:

\section{Quadro I- Agrupamento em Padrões}

\section{Necessidades Psicobiológica}

Paciente, 60 anos, sexo masculino, aposentado, admitido em decorrência de acidente automobilístico, vítima de TCE. Presença de grande ferimento na calota craniana. Admitido na emergência torporoso, mas respondendo ao ser chamado pelo nome. No momento do acidente encontrava-se alerta. Sinais vitais: $P A=$ 90X60MMHG; $R=130 \mathrm{bpm} ; \mathrm{T}=36,2 \mathrm{C}$. Ao exame clínico: pele fria, pálido, pupilas anisocórica, reagente a luz. Encaminhado para o centro cirúrgico para craniotomia e drenagem de hematoma subdural. Admitido na UTI, no pós-operatório imediato de craniotomia. Hospitalizado por 81 dias com seguelas respiratórias e lesões de pele.

Paciente com drenos, sob monitorização da Pressão intra craniana (PIC), não respondendo a estímulos verbais, pupilas anisocóricas (pupila esquerda maior que pupila direita), com sonda nasogastrica (presença de resíduo), com punção venosa pérvia (uso de SF 0,9\%), com sonda vesical de demora, sob respiração mecânica, oximetria de pulso, monitorização cardíaca, presença de úlceras de decúbito e agitação no leito.

\begin{tabular}{|c|c|c|c|c|}
\hline \multicolumn{5}{|c|}{$\begin{array}{l}\text { Quadro II -Raciocínio Diagnóstico segundo RISNER (1990) } \\
\text { Selecionou-se alguns dos diagnósticos de Enfermagem: }\end{array}$} \\
\hline Categorização & Comparação & Inferência & Etiologia & D E \\
\hline $\begin{array}{l}\text { - } 60 \text { anos, sexo } \\
\text { masculino; }\end{array}$ & \begin{tabular}{|lr|}
\multicolumn{3}{|l|}{ Pacientes idosos } \\
apresentam \\
recuperação \\
lenta; estão mais \\
sujeitos & a \\
hematoma & \\
subdural & crônico, \\
que & podem \\
ocorrer & depois \\
de & meses, \\
podendo & ser \\
confundidos & com \\
derrame; r & \\
BRUNNER & $\&$ \\
STUARTH & \\
(1990) &
\end{tabular} & $\begin{array}{l}\text { Risco para } \\
\text { hematoma } \\
\text { subdural crônico } \\
\text { Recuperação } \\
\text { lenta; }\end{array}$ & $\begin{array}{l}\text { - Relacionado ao } \\
\text { TCE; } \\
\text { - Devido à idade } \\
\text { avançada; }\end{array}$ & \begin{tabular}{|lr}
\multicolumn{1}{c}{ Risco } & para \\
enfrentamento & \\
familiar & ineficaz \\
relacionado & com \\
ausência & de \\
responsividade & do \\
paciente, prognóstico \\
imprevisível, & tempo \\
prolongado r & de \\
internação. &
\end{tabular} \\
\hline
\end{tabular}


FEITOZA; Daniela de Souza; FREITAS; Maria Célia de; SILVEIRA; Rita Edna da - Traumatismo crânio- 230 encefálico: diagnósticos de enfermagem a vítimas atendidas em UTI. Revista Eletrônica de Enfermagem, $v$. 06, n. 02, p. 223-233, 2004. Disponível em www.fen.ufg.br

\begin{tabular}{|c|c|c|c|c|}
\hline $\begin{array}{l}\text { Presença de } \\
\text { drenos }\end{array}$ & - Pele integra & $\begin{array}{ll}\text { Risco } & \text { para } \\
\text { infecção; } & \end{array}$ & $\begin{array}{|lr|}\text {-Devido } & \\
\text { presença } & \text { de } \\
\text { drenos, } & \text { lesões } \\
\text { na } & \text { calota } \\
\text { craniana; } & \\
\end{array}$ & $\begin{array}{l}\text {-Risco para infecção } \\
\text { relacionado a } \\
\text { procedimentos } \\
\text { invasivos; }\end{array}$ \\
\hline $\begin{array}{ll} & \text { Não } \\
\text { reagindo a } \\
\text { estímulos } \\
\text { verbais } \\
\text { simples }\end{array}$ & $\begin{array}{l}\text {-Sem } \\
\text { comprometiment } \\
\text { o do centro da } \\
\text { fala }\end{array}$ & \begin{tabular}{|r} 
Déf \\
icit neurológico \\
$-\quad c$ Depressão \\
cerebral;
\end{tabular} & $\begin{array}{|lr|}\text { - } & \text { Relacionado a } \\
\text { lesão } & \text { (torpor, } \\
\text { coma) } & \end{array}$ & \begin{tabular}{|lr}
\multicolumn{2}{c}{ Alteração } \\
sensorial & \\
perceptiva & \\
relacionada & a \\
edema craniano & \\
caracterizado & por \\
ausência & da \\
resposta & a \\
estímulo & \\
\end{tabular} \\
\hline Categorização & Comparação & Inferência & Etiologia & DE \\
\hline $\begin{array}{l}\text { Pupilas } \\
\text { anisocó- } \\
\text { ricas }\end{array}$ & $\begin{array}{l}\text { - Pupilas } \\
\text { simétricas e } \\
\text { reagentes a foco } \\
\text { de luz; }\end{array}$ & $\begin{array}{l}-\quad \text { Risco para } \\
\text { hematoma no } 3^{\circ} \\
\text { nervo craniano }\end{array}$ & 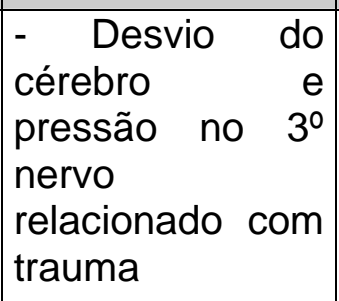 & $\begin{array}{lr}\text { - Perfusão do tecido } \\
\text { cerebral } & \text { alterado } \\
\text { relacionado a edema } \\
\text { caracterizado r por } \\
\text { alteração do estado } \\
\text { mental; } & \end{array}$ \\
\hline $\begin{array}{ll}\text { - } & \text { Sob } \\
\text { ventilação } \\
\text { orotraqueal; }\end{array}$ & $\begin{array}{l}\text { - Padrão } \\
\text { respiratório } \\
\text { normal 16-20 } \\
\text { RPM. } \\
\text { - Tórax: } \\
\text { simétrico; uso da } \\
\text { musculatura } \\
\text { acessória; } \\
\text { ausculta limpa } \\
\text { com murmúrios } \\
\text { vesiculares } \\
\text { normais; }\end{array}$ & $\begin{array}{l}\text { - Retenção de } \\
\text { CO2; } \\
\text { - Hipoventilação; } \\
\text { - Vasodilatação } \\
\text { - Aumento da } \\
\text { PIC; }\end{array}$ & $\begin{array}{l}\text { - Oxigenação } \\
\text { inadequada do } \\
\text { sangue } \\
\text { circulante; }\end{array}$ & $\begin{array}{lr}\text {-Padrão respiratório } \\
\text { ineficaz relacionado a } \\
\text { lesãor } & \text { cerebral } \\
\text { caracterizado por } & \text { papacidader vital } \\
\text { caminuída; } & \\
\text { dimina } & \text {-Desobstrução } \\
\text { ineficaz das vias } \\
\text { aéreas relacionadas } \\
\text { com hipóxia } \\
\text { caracterizado pela } \\
\text { incapacidade eliminar } \\
\text { secreções; }\end{array}$ \\
\hline
\end{tabular}


FEITOZA; Daniela de Souza; FREITAS; Maria Célia de; SILVEIRA; Rita Edna da - Traumatismo crânio- 231 encefálico: diagnósticos de enfermagem a vítimas atendidas em UTI. Revista Eletrônica de Enfermagem, $v$.

06, n. 02, p. 223-233, 2004. Disponível em www.fen.ufg.br

\section{Planejamento das ações de Enfermagem}

a) DE- Risco para enfrentamento familiar ineficaz relacionado com ausência de responsividade do paciente.

Meta: familiares desenvolvam mecanismos de enfrentamento adaptativos;

Objetivo: enfrentar a situação de ausência de ente querido;

- Intervenções de Enfermagem:

Manter a família informada do estado do paciente - em todos os horários de visita Incentivar a espiritualidade solicitar a presença de líder religioso

Implementação:

Durante os horários de visita, a família demonstrava preocupada com a rápida recuperação. $\mathrm{O}$ paciente representa a única fonte de renda.

Orientado quando ao tempo necessário para a recuperação e o tratamento necessitar ser prolongado.

Avaliação

Durante todas as visitas a família mantinha a mesma preocupação e não aceitava a necessidade da demora do tratamento.

b) DE- Risco para infecção relacionados a procedimentos invasivos;

Meta: Ausência de infecção

Objetivo: Evitar aparecimento de processos infecciosos, traumas

- Intervenções de Enfermagem:

-Realizar procedimento

obedecendo a técnicas

assépticas - quando

necessário

- Lavagem das mãos

- Observar sinais vitais;

- Higiene geral- $M-T-N$

Implementação

Observar a higiene
rigorosamente nos
realizados. Comorios
cabeludo, bucal e presença de
flebites. Renovado sonda
vesical ao final da primeira
semana. Durante a realização
dos curativos, observados
sinais flogísticos.

Avaliação

Ao final das doze horas paciente mostrava-se com aspecto limpo sem temperatura elevado em nenhum período. Mantendo conduta de enfermagem quando ao diagnóstico de risco de infecção

c) DE Alteração sensorial perceptiva relacionada a edema craniano caracterizado por ausência de reação pupilar;

Meta: Recuperação da percepção sensorial;

Objetivo: Reagir aos estímulos sensoriais

- Intervenções:

-Solicitar avaliação

neurológica;;

-Manter diálogo com o

paciente durante

procedimentos; Sempre que

necessário

- Administrar analgésicos que

não mascarem o nível de consciência; ao sinal de dor

- Aplicar compressas frias nos olhos; $M$ - $T$ - N;

Implementação

Após avaliação neurológica realizado, percebeu-se a não reação pupilar a luz. Entrouse em contato com o neurologista de plantão.

Como paciente mantinha olhos abertos, orientado a equipe que durante a higiene 
FEITOZA; Daniela de Souza; FREITAS; Maria Célia de; SILVEIRA; Rita Edna da - Traumatismo crânio- 232 encefálico: diagnósticos de enfermagem a vítimas atendidas em UTI. Revista Eletrônica de Enfermagem, v.

06, n. 02, p. 223-233, 2004. Disponível em www.fen.ufg.br

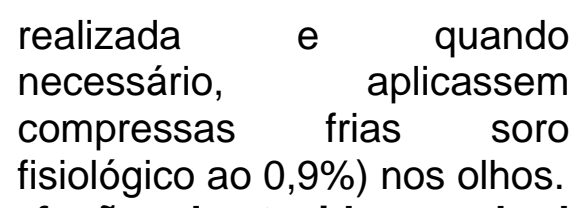

d) DE Perfusão do tecido cerebral alterada: menor que as necessidades corporais relacionada a inabilidade para ingerir e deglutir caracterizada pela presença de trauma;

Meta: Melhora da homeostasia neurológica;

Objetivos: Incentivar quando consciente a respiração espontânea;

- Intervenções:

- Elevar a cabeceira a 30 graus- Contínua

- Controlar os níveis da PICquando instalado

- Avaliar a gasometriaquando saturação menos que $85 \%$

Implementação:

Durante o plantão manteve-se contínua observação quanto aos níveis de saturação. Observou-se ainda a necessidade de manter cabeceira elevada, principalmente durante as gavegens. Retirado aparelho de controle do PIC com 72 horas de internamento na unidade.

\section{Avaliação}

Ao final do plantão de doze hora, paciente continuava sem reação pupilar a luz e manteve-se os diagnósticos de enfemagem.

e) DE Padrão respiratório ineficaz relacionado a lesão cerebral caracterizado por capacidade vital diminuída;

Meta: Monitorizar padrões respiratórios a níveis normais;

Objetivo: Alcançar níveis respiratórios satisfatórios;
- Intervenções:

- Aspiração cautelosa traquéia

e faringe - Sempre que

necessário

- Manter ventilação controlada

- Observar os alarmes

- Ausculta de sons

respiratórios; $M T N$

- Monitorizar respiração

mecânica - Contínua

\section{Implementação}

No início do plantão realizouse avaliação do paciente e detectado a necessidade de realizar aspiração traqueobronquica. Presença se secreção esbranquiçada e fluida. Ausculta sem ruídos.

\section{Avaliação}

Ao final do plantão o paciente manteve saturação em torno de $95 \%$ e moderada quantidade de secreção.

\section{CONSIDERAÇÕES FINAIS}

Através da realização da pesquisa, buscamos identificar o tipo de trauma mais prevalente nas UTIs e a partir daí elaborar um modelo de implantação dos diagnósticos de enfermagem, a partir da taxonomia de NANDA I. O uso do raciocínio de Risner facilitou o raciocínio clínico, bem como a identificação do principais diagnósticos de enfermagem para o paciente em estudo.

O desenvolvimento desta pesquisa favoreceu a identificação de um perfil dos diagnósticos de Enfermagem a pacientes vítimas de TCE e proporcionou a elaboração de um modelo de implantação para aquela unidade.

Esse processo de análise levou ao aprofundamento da assistência a pacientes vítimas de trauma, especialmente ao doente crítico vítima de TCE.

Procurou-se estabelecer os principais diagnósticos de enfermagem, que ajudariam os 
FEITOZA; Daniela de Souza; FREITAS; Maria Célia de; SILVEIRA; Rita Edna da - Traumatismo crânio- 233 encefálico: diagnósticos de enfermagem a vítimas atendidas em UTI. Revista Eletrônica de Enfermagem, $v$.

06, n. 02, p. 223-233, 2004. Disponível em www.fen.ufg.br

enfermeiros que lidam diretamente com essa clientela a planejar a assistência, otimizando o tempo e garantindo qualidade no atendimento.

Desse modo o planejamento das ações através do uso do raciocínio clínico, do processo de enfermagem a pacientes críticos possibilita um melhor direcionamento da assistência de enfermagem, otimizando tempo e garantindo uma assistência de qualidade.

\section{REFERÊNCIAS BIBLIOGRÁFICAS}

LUDKE, M. E. D. A.; ANDRE, D. A; Pesquisa em educação: Abordagens qualitativas. São Paulo: EPU, 1996.

ARRUDA, A. J. GARCIA, Telma L. R. Diagnóstico de Enfermagem relacionados a oxigenação, atribuídos a vítimas de traumas admitidos em CTI. Rev. Bras. Enf., v 53, n 3. p 368-371. 2000.
BRUNNER, L.S; SUDDARTH D.S et al. Assistência a pacientes com distúrbios neurológicos. Tratado de Enfermagem médico cirúrgica. $6^{\mathrm{a}}$ ed. Rio de Janeiro: Guanabara Koogan, 1990

CINTRA, E. A . Assistência de enfermagem ao paciente crítico. São Paulo: Atheneu, 2000.

PEIXOTO, Maria das Graças Barbosa. Estudo Epidemiológico das vítimas de acidentes de trânsito admitidas em hospital de emergência em Fortaleza. Fortaleza, $1998.93 \mathrm{fls}$. Dissertação (Mestrado) Universidade Federal do Ceará.

RISNER, P.B. Nursing diagnosis: diagnostic sistements. In: Christensen, P. J; Kenney, J.W. ed. Nursing Process: application of conceptual modes. $3^{\mathrm{a}}$ ed. St. Louis, Mosby, 1990.

Texto original recebido em 13/06/2004

Publicação aprovada em31/08/2004 